\begin{tabular}{|l|l|l|l|l|}
\hline Cuadernos de Investigación Geográfica & 2003 & $N^{\circ} 29$ & pp. 23-34 & ISSN 0211-6820 \\
\hline
\end{tabular}

(C) Universidad de La Rioja

\title{
APLICACIÓN DE LAS TABLAS DE CONTINGENCIA (CROSS-TAB ANALYSIS) AL ANÁLISIS ESPACIAL DE TENDENCIAS CLIMÁTICAS EN EL SECTOR ORIENTAL DE LA PENÍNSULA IBÉRICA
}

\author{
MARTÍN DE LUÍS (1) \\ SERGIO M. VICENTE (2) \\ JOSÉ CARLOS GONZÁLEZ HIDALGO (2)(*) \\ JOSÉ RAVENTÓS (1)
}

(1) Departamento de Ecología, Universidad de Alicante, 03080, Alicante, España

(2) Departamento de Geografía, Universidad de Zaragoza, 50009, Zaragoza, España

(*)jcgh@posta.unizar.es

\begin{abstract}
RESUMEN: Proponemos el análisis de las tablas cruzadas (cross-tab analysis) para estudiar el solapamiento y analizar las relaciones espaciales entre las tendencias de diferentes variables del clima. Para ilustrar el método se presentan dos ejemplos en los que se comparan las distribuciones espaciales de las tendencias anuales de la precipitación, del evento diario máximo y de la variabilidad anual. Los ejemplos se desarrollan en la Comunidad Valenciana y el sector central de la Depresión del Ebro. En la Comunidad Valenciana el análisis permite descubrir una relación doble entre las tendencias de los totales anuales y las del máximo evento diario. Por su parte, en el sector central del valle del Ebro la relación encontrada entre las tendencias de precipitación anual y su variabilidad sugiere que los escenarios futuros, si se mantienen las pautas descritas, estarán sometidos a una mayor imprecisión y aleatoriedad.

ABSTRACT: We suggest cross-tab analysis to study the spatial distribution-overlapping trend between climate elements. We present two examples of such overlapping, in which we compared the spatial distribution of annual rainfall trend with spatial distribution of trend of maximum daily event and spatial distribution of annual rainfall variability. In the first ones (Comunidad de Valencia, E of Spain) we find a complex relationship between annual rainfall trend and daily maximum rainfall trend. In the second ones (Depresión del Ebro, NE inland of Spain) the relationship between annual rainfall trend and annual rainfall variability trend suggest that futures scenarios could be more and more non-predictable.
\end{abstract}

Palabras clave: Tendencias climáticas; tablas de contingencia; análisis espacial; Comunidad Valenciana; depresión del Ebro.

Key words. Climatic trends; cross tab analysis; spatial analysis; Valencia Region; Ebro basin. 


\section{Introducción}

El análisis de la evolución temporal de los elementos climáticos es complejo. En el caso de las precipitaciones la evolución de los totales anuales no tiene por qué ocurrir de la misma forma que la de los valores diarios, mensuales o estacionales. Por tanto se necesita establecer métodos que permitan identificar las interrelaciones entre las tendencias de los distintos componentes y las tendencias del patrón general para, por ejemplo, conocer si existen relaciones entre las tendencias de los valores anuales y las de una determinada estación, un mes o un tipo de evento.

En general en los estudios climáticos los resultados espaciales del análisis temporal se presentan en forma de mapas de signos de tendencias en diversas escalas temporales (véase un reciente ejemplo en Klein et al. 2002). Karl y Knight (1998), Easterling et al. (2000) y Brunetti et al. (2001), entre otros muchos, han señalado la coincidencia entre el aumento anual de las precipitaciones y el aumento del volumen de los eventos extremos, analizando las variaciones estacionales de sus tendencias. En otros casos se ha mostrado la diferente distribución espacial de las tendencias estacionales respecto a la de los totales anuales (véase p.e. González Hidalgo et al., 2001). Sin embargo las conclusiones de estos trabajos se fundamentan en el análisis aislado de cada variable analizada (tendencia estacional, diaria, etc) sin considerar las posibles correspondencias locales de los distintos componentes que contribuyen a la evolución del total anual. Por tal razón, el solape de documentos espaciales de signos de tendencias necesita ser sometido a un análisis que confirme la aleatoriedad o asociación de los signos de tendencia.

En el presente trabajo proponemos aplicar la técnica denominada tabla de contingencia al análisis de las distribuciones espaciales de datos categóricos, particularmente a los signos de tendencias de las precipitaciones. Este método permite identificar estadísticamente si los patrones temporales observados en la tendencia de una variable se puede asociar con la evolución de otras, y así establecer modos de comportamiento.

Para ilustrar la aplicación de la técnica, mostraremos dos casos en los que se relacionan la distribución espacial de las tendencias anuales de precipitación con la distribución de las tendencias de los eventos extremos y la variabilidad de la precipitación anual. La razón fundamental para elegir las precipitaciones como elemento de análisis es su elevada variabilidad en el tiempo y el espacio, a la vez que es el factor determinante que controla los sistemas naturales en los ambientes de clima mediterráneo.

\section{El estudio de la asociación espacial de los signos de tendencias}

Para solapar la distribución espacial de las tendencias proponemos el empleo de tablas cruzadas (tablas de contingencia) considerando que "los datos aleatoriamente seleccionados" son los observatorios meteorológicos y la "característica" evaluada es el signo de tendencia de analizado (positivo, negativo o no significativo). En realidad el registro localizado de los datos climáticos obliga a este planteamiento, en tanto la medida no se realiza aleatoriamente en el espacio, sino que proviene del lugar en donde están instalados los instrumentos registradores. 
En el caso que nos ocupa, puesto que las tendencias solamente pueden ser positivas, no significativas y negativas ( $\mathrm{p}<0.05$, véase más adelante), la estructura de las tablas de contingencia es $3 \times 3$ (filas x columnas). Una vez construida la tabla de cruce esta se puede manipular como una matriz de transición de frecuencias. Exponemos a continuación un ejemplo basado en dos distribuciones aleatorias indicadas en la Tabla 1.

\begin{tabular}{|c|c|c|c|c|c|c|c|c|c|c|c|c|c|c|c|c|c|c|c|c|c|c|c|c|c|c|c|c|c|}
\hline \multicolumn{30}{|c|}{$\begin{array}{l}\text { Tabla 1.- Distribuciones aleatorias de signos }(1,0) \text { y solapamiento entre ambas. La distribución A y B se han obtenido } \\
\text { aleatoriamente, y para ilustrar el solapamiento final, en la distribución B se han hecho coincidir los } 20 \text { primeros regis- } \\
\text { tros (columnas iniciales de la izquierda). El resultado del test de } \chi \text { es de } 17.90(p=0,0001) \text {, el valor del CC } 0,39, \text { que } \\
\text { representa sobre el CC } \max \text { un valor de } 0,48 \% . \\
\text { * Códigos de solapamiento: }(1,1=1),(1,0=2),(1,-1=3),(0,1=4),(0,0=5),(0,-1=6),(-1,1=7),(-1,0=8),(-1,1=9)\end{array}$} \\
\hline \multicolumn{10}{|c|}{ DISTRIBUCIÓN A } & \multicolumn{10}{|c|}{ DISTRIBUCIÓN B } & \multicolumn{10}{|c|}{ SOLAPAMIENTOS } \\
\hline-1 & 0 & & 0 & & & 1 & & & & \begin{tabular}{|l|}
-1 \\
\end{tabular} & & & & 1 & & $\mid-1$ & & & & \begin{tabular}{|l|}
9 \\
\end{tabular} & 5 & 7 & 4 & 1 & \begin{tabular}{|l|}
2 \\
\end{tabular} & 3 & & & \\
\hline 1 & 0 & 0 & -1 & $\frac{1}{1}$ & 1 & \begin{tabular}{|l|} 
\\
\end{tabular} & -1 & & \begin{tabular}{|l|} 
\\
\end{tabular} & \begin{tabular}{|l|}
1 \\
\end{tabular} & 0 & \begin{tabular}{|l|}
-1 \\
\end{tabular} & 1 & 1 & 0 & $\mid-1$ & 1 & 0 & 1 & 1 & 5 & 6 & 7 & 1 & \begin{tabular}{|c|}
2 \\
\end{tabular} & 9 & & 5 & \\
\hline 1 & & & 1 & & & 1 & & & $\left.\right|^{-1}$ & & & & & 1 & -1 & $\begin{array}{l}0 \\
\end{array}$ & -1 & 0 & \begin{tabular}{|l|} 
\\
\end{tabular} & & & & & 4 & \begin{tabular}{|l|}
9 \\
\end{tabular} & & & 2 & 9 \\
\hline 0 & 1 & & 1 & 4 & -1 & 1 & -1 & 0 & \begin{tabular}{|l|l|} 
\\
\end{tabular} & 0 & 1 & & 1 & 0 & 1 & $\mid-1$ & \begin{tabular}{|l|} 
\\
\end{tabular} & -1 & 1 & & 1 & 5 & 1 & 8 & \begin{tabular}{|l|}
7 \\
\end{tabular} & 3 & 9 & 6 & 4 \\
\hline 0 & -1 & & 0 & -1 & -1 & 0 & -1 & 0 & 1 & \begin{tabular}{|l|}
0 \\
\end{tabular} & -1 & 1 & 1 & 0 & 0 & $|-1|$ & \begin{tabular}{|l|}
0 \\
\end{tabular} & 1 & 1 & 5 & 9 & 4 & 4 & 8 & \begin{tabular}{|l|}
8 \\
\end{tabular} & 0 & 8 & 4 & 1 \\
\hline 1 & -1 & & 0 & -1 & -1 & 0 & & -1 & \begin{tabular}{|l|} 
\\
\end{tabular} & & & & & 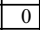 & & 1 & & 1 & \begin{tabular}{|l|}
-1 \\
\end{tabular} & & & & & 8 & \begin{tabular}{|l|}
9 \\
\end{tabular} & & & 9 & 9 \\
\hline-1 & 1 & 0 & 0 & 1 & \begin{tabular}{|l|}
1 \\
\end{tabular} & \begin{tabular}{|l|} 
\\
\end{tabular} & 0 & 0 & 1 & \begin{tabular}{|l|}
-1 \\
\end{tabular} & -1 & 0 & -1 & 0 & -1 & $\begin{array}{l}0 \\
\end{array}$ & 1 & -1 & 1 & \begin{tabular}{|c|}
9 \\
\end{tabular} & 9 & 2 & 9 & \begin{tabular}{|l|}
8 \\
\end{tabular} & \begin{tabular}{|l|}
6 \\
\end{tabular} & 2 & \begin{tabular}{|l|}
4 \\
\end{tabular} & 6 & 7 \\
\hline-1 & 1 & 0 & 0 & 1 & 1 & $\mid-1$ & 1 & -1 & 1 & \begin{tabular}{|l|}
-1 \\
\end{tabular} & 1 & -1 & 0 & 1 & 1 & 1 & 0 & -1 & 1 & & 1 & 6 & 3 & 1 & \begin{tabular}{|l|}
1 \\
\end{tabular} & 7 & 2 & 9 & 1 \\
\hline 0 & 0 & & 0 & 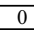 & 0 & -1 & -1 & 0 & 1 & 0 & & & & 0 & -1 & 1 & -1 & 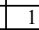 & 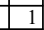 & & 5 & & 4 & 5 & \begin{tabular}{|l|}
6 \\
\end{tabular} & 7 & 9 & 4 & 1 \\
\hline 0 & -1 & 0 & 0 & & & 0 & & 0 & \begin{tabular}{|l|} 
\\
\end{tabular} & \begin{tabular}{|l|}
0 \\
\end{tabular} & -1 & & -1 & \begin{tabular}{|l|}
-1 \\
\end{tabular} & & 1 & & & 1 & \begin{tabular}{|c|}
5 \\
\end{tabular} & 9 & 6 & 6 & \begin{tabular}{|l|}
3 \\
\end{tabular} & \begin{tabular}{|c|}
5 \\
\end{tabular} & 4 & & & 1 \\
\hline
\end{tabular}

Paso 1. Confección de la tabla de cruce siguiendo los resultados de la Tabla 1 (distribuciones A y B).

\begin{tabular}{|c|c|c|c|c|c|}
\hline & \multicolumn{3}{|c|}{ A } & \multirow[b]{2}{*}{ Total } \\
\hline & & + & $\mathrm{ns}$ & - & \\
\hline \multirow{3}{*}{$\infty$} & + & 19 & 11 & 10 & 40 \\
\hline & ns & 8 & 14 & 6 & 28 \\
\hline & - & 3 & 11 & 18 & 32 \\
\hline & Total & 30 & 36 & 34 & 100 \\
\hline
\end{tabular}

Paso 2. Al dividir cada frecuencia particular por el total de la fila correspondiente, se obtiene la probabilidad de cambio de un signo a otro en el orden expresado en cada cruce. Esta información además permite construir un diagrama de flujos que puede ayudar a elucidar la naturaleza de los cambios entre las tendencias comparadas.

\begin{tabular}{|c|c|c|c|c|}
\hline & \multicolumn{3}{|c|}{ A } \\
\hline & & + & ns & - \\
\hline \multirow{3}{*}{$\infty$} & + & 0.475 & 0.275 & 0.250 \\
\hline & ns & 0.286 & 0.500 & 0.214 \\
\hline & - & 0.094 & 0.344 & 0.563 \\
\hline
\end{tabular}

Paso 3. Se calcula el vector de probabilidad dividiendo el total de cada columna de la matriz de transición de frecuencias por el número de observaciones, esto es obtenemos la probabilidad que tiene cada signo de tendencia de cambiar si la probabilidad es independiente de dicho signo. 


\begin{tabular}{|c|c|c|c|c|}
\hline & \multicolumn{3}{|c|}{$\mathrm{A}$} \\
\hline & & + & ns & - \\
\hline \multirow{3}{*}{ 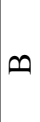 } & + & 0.300 & 0.360 & 0.340 \\
\hline & ns & 0.300 & 0.360 & 0.340 \\
\hline & - & 0.300 & 0.360 & 0.340 \\
\hline
\end{tabular}

Paso 4. Al multiplicar dichos valores por los totales de cada fila obtenemos de las frecuencias observadas la matriz aleatoria de frecuencias esperadas.

\begin{tabular}{|l|l|lll|}
\cline { 3 - 5 } \multicolumn{2}{c|}{} & \multicolumn{3}{c|}{ A } \\
\cline { 3 - 5 } \multicolumn{1}{c|}{} & + & $\mathrm{ns}$ & - \\
\hline \multirow{4}{*}{$\curvearrowleft$} & + & 12.0 & 14.4 & 13.6 \\
& $\mathrm{~ns}$ & 8.4 & 10.1 & 9.5 \\
& - & 9.6 & 11.5 & 10.9 \\
\hline
\end{tabular}

Paso 5. La comparación de las frecuencias observadas y esperadas se realiza mediante la prueba de $\chi^{2}$.

En general la hipótesis nula $\left(\mathrm{H}_{0}\right)$ es que los datos proceden de una población cuyas transiciones entre signos son aleatorias. En los ejemplos desarrollados a continuación dicha hipótesis será "que la distribución de las tendencias anuales no se solapa con la distribución de la tendencia comparada". Como hipótesis alternativa $\left(\mathrm{H}_{1}\right)$ hemos considerado en todos los casos que las tendencias comparadas proceden de una población de transiciones que no son aleatorias, es decir que existe asociación espacial con el signo de la tendencia de la precipitación anual. Los grados de libertad asignados son en todos los casos $(\mathrm{m}-1)^{2}$, cuya probabilidad hemos evaluado mediante modelos de Montecarlo con prueba de dos colas en el programa estadístico SPSS ${ }^{\mathrm{TM}}$. Detalles del procedimiento y aplicación a otros ámbitos de estudio de los sistemas naturales se encuentran detallados en Swan y Sandilans (1995, p. 215-221).

Como la prueba descrita solamente establece si existe, o no, una asociación entre las variables comparadas, cuando la hipótesis alternativa $\left(\mathrm{H}_{1}\right)$ se confirma ésta no proporciona una medida de la fortaleza de la misma. En estos casos el grado de la asociación se puede evaluar por medio del Coeficiente de Contingencia (CC) (Clark y Hosking, 1986, p. 265-266), de la manera siguiente:

$$
\mathrm{CC}=\sqrt{\frac{\chi^{2}}{\mathrm{n}+\chi^{2}}}
$$

con (n) la frecuencia absoluta. Dicho estadístico no asume ninguna distribución previa de la variable (Downie y Heath, 1986, p. 246), y su máximo valor se estima del siguiente modo (Sachs, 1978, p. 402-403): 


$$
\mathrm{CC}=\sqrt{\frac{\mathrm{K}-1}{\mathrm{~K}}}
$$

que en el caso que nos ocupa, con tablas de estructura $(3 \times 3)$, es $0.82(\mathrm{~K}=$ filas $)$. En cada caso a su vez estimamos el valor de la ratio $\mathrm{CC} / \mathrm{CC}_{\max }$ que permite comparar situaciones distintas.

Por último, para caracterizar los diferentes solapamientos entre las tendencias analizadas se comprueba la simetría de la matriz observada mediante la prueba de Bowker (véase Sachs, 1978, p. 407-408). En este caso la hipótesis nula $\left(\mathrm{H}_{0}\right)$ es que el solape de las distribuciones observadas es simétrica, según

$$
B_{i j}=B_{j i}
$$

con $\mathrm{B}_{\mathrm{ij}}=$ la frecuencia observada en la i-ésima fila con $\mathrm{j}$-ésima columna, $\mathrm{y}_{\mathrm{ji}}=$ la frecuencia observada en la j-ésima fila con i-ésima columna, estimando un nuevo valor de $\chi_{\text {sim }}^{2}$ del siguiente modo:

$$
\chi^{2} \operatorname{sim}=\sum_{j=1}^{r-1} \sum_{i>j}=\frac{\left(B_{i j}-B_{j i}\right)^{2}}{B_{i j}+B_{j i}}
$$

con grados de libertad según [(K (K-1)) / 2], de nuevo con el valor de probabilidad 0.05 y $\mathrm{K}=$ filas. Como hipótesis alternativa $\left(\mathrm{H}_{1}\right)$ se considera la asimetría de la matriz.

Por último, la interpretación final de los solapamientos se puede realizar combinando el análisis de independencia, las matrices de transición de probabilidad empírica, la fortaleza de la asociación y la simetría.

\section{Aplicación y desarrollo}

Para ilustrar el método propuesto se han desarrollado dos ejemplos aplicados en dos ambientes diferenciados. En el primero de ellos, la Comunidad Valenciana, se comparan las tendencias de la precipitación anual y del máximo diario. El caso ilustra el estudio de las condiciones de torrencialidad de las precipitaciones de la costa mediterránea de la Península Ibérica y la dependencia del total anual de un bajo número de eventos (el máximo en este caso). En segundo lugar se analiza la relación entre las tendencias del volumen anual y de su variabilidad en el sector central de la Depresión del Ebro, como exponentes de la aleatoriedad de las precipitaciones en ambientes mediterráneos.

\subsection{Bases de datos}

Se han empleado dos bases de datos distintas en su origen que en el momento actual están siendo combinadas entre sí y ampliadas (González Hidalgo et al., 2002a; González Hidalgo et al. 2002b). 
En el primer caso los datos proceden del Atlas Climático de la Comunidad Valenciana. La base de datos original consta de datos diarios de 97 observatorios y abarca el período 1951-1990, cubriendo al menos el normal 1961-1990 (fuente original Pérez Cueva, 1994). Anualmente en cada observatorio se seleccionó el máximo evento diario que representa un promedio en torno al 7-10 \% de la precipitación total anual (de Luís, 2000). En el segundo caso la base de datos se compone de 73 observatorios localizados en el sector central de la depresión del Ebro y cubren el período 1971-2000. La densidad promedio de observatorios en las dos bases de datos se sitúa en torno a 1 observatorio cada $200 \mathrm{~km}^{2}$.

En ambos casos, tras comprobar la homogeneidad de las series (test de Barlett y SNHT) se aplicó un filtro de paso bajo ( 9 y 5 años respectivamente a los datos procedentes de la Comunidad Valenciana y de la Depresión del Ebro) a las series de totales anuales, del máximo anual, de la contribución del máximo anual como porcentaje y se calcularon los coeficientes de variación móviles con una ventana de 5 años en las series de la depresión del Ebro. La evaluación de tendencias significativas en el tiempo se estimó mediante el coeficiente de correlación no paramétrico rho de Spearman, con $\mathrm{p}<0.05$.

\subsection{Ejemplo 1}

\section{Relaciones entre la tendencia de precipitación anual y el máximo de precipitación diario en la Comunidad Valenciana.}

La Tabla 2 indica el número de observatorios de la Comunidad Valenciana según el signo de tendencia de la precipitación anual, de la tendencia del evento extremo en volumen y de la proporción que dicho evento extremo supone en porcentaje sobre el total anual en el período 1961-1990 (de Luís, 2000).

\begin{tabular}{|c|c|c|c|}
\hline $\begin{array}{r}\text { Tabla 2.- Número de observat } \\
\text { el signo de tendencia de la } \\
\text { como porcentaj }\end{array}$ & $\begin{array}{l}\text { torios en } \\
\text { recipitac } \\
\text { e de la p }\end{array}$ & $\begin{array}{l}\text { la Comun } \\
\text { ión anual, } \\
\text { ecipitació }\end{array}$ & $\begin{array}{l}\text { idad Valen } \\
\text { del máxi } \\
\text { n anual. }\end{array}$ \\
\hline Tendencia & P. anual & Máximo & $\begin{array}{l}\text { Máximo } \\
\text { (como \%) }\end{array}$ \\
\hline Positiva & 11 & 35 & 44 \\
\hline No significativa & 42 & 47 & 24 \\
\hline Negativa & 44 & 15 & 29 \\
\hline
\end{tabular}

La Figura 1 muestra la distribución espacial de dichas tendencias. En la Comunidad Valenciana durante el período 1961-1990 han predominado los signos negativos de la tendencia anual (Figura 1 a), así como los de valor positivo en el máximo diario, tanto en volumen (Figura 1 b) como en porcentaje de contribución (Figura 1 c) (de Luís, 2000; de Luís et al., 2000). 


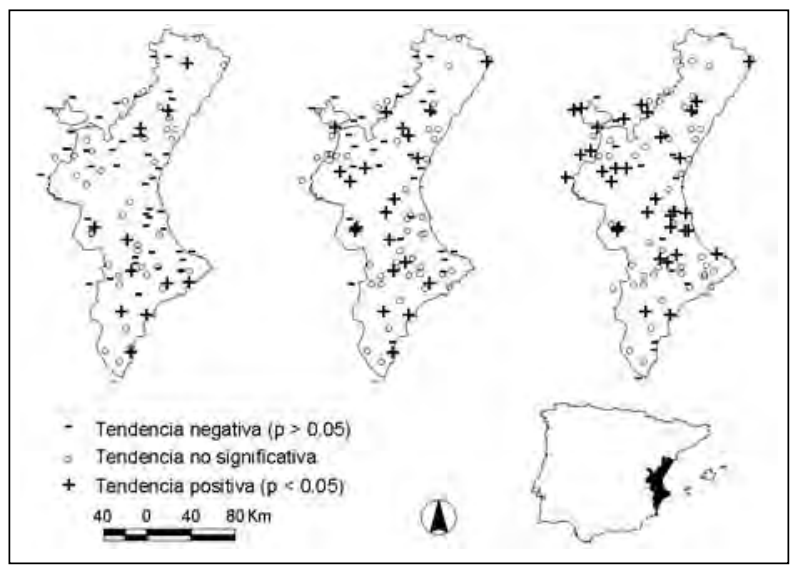

Figura 1.- Distribución espacial de la tendencia de precipitación anual (a), del volumen del máximo diario (b) y del máximo como porcentaje anual (c) en la Comunidad Valenciana.

La matriz de cruce entre la tendencia anual y máximo diario $(\mathrm{mm})$ se muestra en la Tabla 3, acompañada de la probabilidad de transición empírica (p.t.e)

\begin{tabular}{|c|c|c|c|c|c|c|c|c|c|c|}
\hline $\begin{array}{r}\text { Tabl } \\
\text { en }\end{array}$ & 3.- & & enct & de lo & $\begin{array}{l}\text { prec } \\
\text { cian }\end{array}$ & $\begin{array}{l}\text { ión } \\
e .: p\end{array}$ & $\begin{array}{l}\text { nual } \\
\text { obab }\end{array}$ & $\begin{array}{l}y \text { del mo } \\
\text { ilidad }\end{array}$ & $\begin{array}{l}\text { áximo e } \\
\text { e transi }\end{array}$ & $\begin{array}{l}\text { vento ( } \\
\text { ción em }\end{array}$ \\
\hline & & & & & & encis & anu & & & \\
\hline & & + & $\mathrm{ns}$ & - & & & & + & $\mathrm{ns}$ & - \\
\hline & + & 9 & 13 & 0 & 16 & & + & 0.409 & 0.591 & 0.000 \\
\hline$\stackrel{̊}{g}$ & ns & 2 & 21 & 22 & 42 & $0^{\circ}$ & ns & 0.044 & 0.467 & 0.489 \\
\hline$\sum^{-\pi}$ & - & 0 & 8 & 22 & 39 & 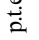 & - & 0.000 & 0.267 & 0.733 \\
\hline & & & 42 & 44 & 97 & & & & & \\
\hline
\end{tabular}

En la Tabla 4 se expone la distribución de frecuencias observadas entre la tendencia del volumen anual y la tendencia del porcentaje de contribución del máximo anual.

$$
\begin{aligned}
& \text { Tabla 4. Tendencia de la precipitación anual (volumen) y del máximo evento (como } \\
& \text { porcentaje) en la Comunidad Valenciana. P.t.e.: probabilidad de transición empírica. } \\
& \text { Tendencia anual }
\end{aligned}
$$

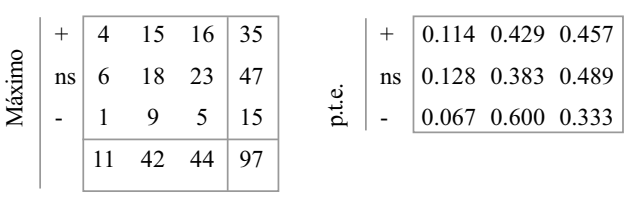

Los resultados de las diversas pruebas estadísticas se muestran en la Tabla 5. 


\begin{tabular}{|c|c|c|c|c|c|c|c|}
\hline \multicolumn{8}{|c|}{$\begin{array}{c}\text { Tabla 5. Resultados de las pruebas de independencia y simetría entre las tendencias } \\
\text { del volumen anual y del máximo diario. Comunidad Valenciana. Se incluye el CC y } \\
\text { su porcentaje sobre el máximo (\% CCmax). Los signos (/,V indican la diagonal } \\
\text { principal sobre la que se aplica la prueba de simetría, que nos indica la dirección } \\
\text { predominante del cambio. n.s.: no significativo. A(vol) es la precipitación anual en } \\
\text { volumen; Max (vol) es la precipitación del máximo anual por volumen; Max (\%) } \\
\text { idem en porcentaje sobre el total anual. }\end{array}$} \\
\hline \multirow[b]{3}{*}{ Comparación } & \multicolumn{4}{|c|}{ Independencia } & \multicolumn{3}{|c|}{ Simetría } \\
\hline & \multicolumn{4}{|c|}{ g.1. $(3-1)^{2}$, crit. 9.48} & \multicolumn{3}{|c|}{ g.1. $(3 \times(3-1)) / 2$, crit. 7.815} \\
\hline & ${ }_{-}^{2}$ & $\mathrm{p}$ & $\mathrm{CC}$ & $\% \mathrm{CC}_{\max }$ & $2^{2}$ & $\mathrm{p}$ & Diagonal \\
\hline $\mathrm{A}(\mathrm{vol})-\mathrm{Max}(\mathrm{vol})$ & 40.83 & 0.000 & 0.544 & 0.663 & 11.40 & 0.05 & $\backslash$ \\
\hline $\mathrm{A}(\mathrm{vol})-\mathrm{Max}(\%)$ & 2.22 & n.s. & - & - & 2.40 & n.s. & / \\
\hline
\end{tabular}

La relación espacial de los signos de tendencia del volumen anual y del máximo diario anual se muestra significativa $(\mathrm{p}<0.001)$, y la fortaleza de dicha asociación es elevada $\left(\% \mathrm{CC}_{\max }=0.663\right)$, mientras no ocurre así entre la tendencia del volumen y de la proporción del máximo anual, cuya asociación resulta ser no significativa y por tanto de carácter aleatorio.

Las pruebas de simetría refuerzan los resultados precedentes y presentan una situación compleja. La relación entre el signo de tendencia anual y del evento máximo no es simétrica, pero las relaciones de los signos son directas. Por el contrario la relación entre la tendencia anual y del evento máximo como porcentaje ofrece una transición simétrica, y la dirección predominante es indirecta.

Las probabilidades de transición entre las tendencias evaluadas por volumen son sumamente sugerentes. El cruce de signos doblemente negativo agrupa 22 observatorios (1/4 aproximadamente del total), con una elevada p.t.e. (0.733). Otras transiciones que se manifiestan con elevada p.e.t. son los cruces (-/ns) y (ns/ns) que agrupan 42 casos, casi el $50 \%$ de observatorios.

Esta relación en principio parece sugerir que la precipitación anual pudiera depender del evento máximo diario de cada año, pero el carácter aleatorio de la asociación de tendencias anuales y del porcentaje del máximo indica que tal conclusión debe ser ponderada con cautela, y aunque la asociación de tendencias de los valores absolutos existe, la ausencia de solapamiento significativo permite sugerir que el comportamiento espacial de los eventos máximos anuales no es uniforme y presenta una gran variabilidad de situaciones.

\subsection{Ejemplo 2}

\section{Relaciones entre la tendencia anual y la tendencia de la variabilidad anual en el sec- tor central de la depresión del Ebro.}

En la Tabla 6 se muestra el número de observatorios según el signo de tendencias de la precipitación anual y de su variabilidad en el sector central de la depresión del Ebro. 


\begin{tabular}{|c|c|c|}
\hline \multicolumn{3}{|c|}{$\begin{array}{l}\text { Tabla 6.- Número de observatorios en el sector central de la depresión } \\
\text { del Ebro según el signo de tendencia de la precipitación anual y de la } \\
\text { tendencia del coeficiente de variación anual móvil. }\end{array}$} \\
\hline Tendencia & P. anual & Variabilidad \\
\hline Positiva & 2 & 25 \\
\hline No significativa & 31 & 40 \\
\hline Negativa & 38 & 2 \\
\hline
\end{tabular}

Su distribución se muestra en las Figura 2. En general predominan las tendencias de precipitación de signo negativo durante los últimos treinta años del siglo XX, y la variabilidad ha sido creciente.

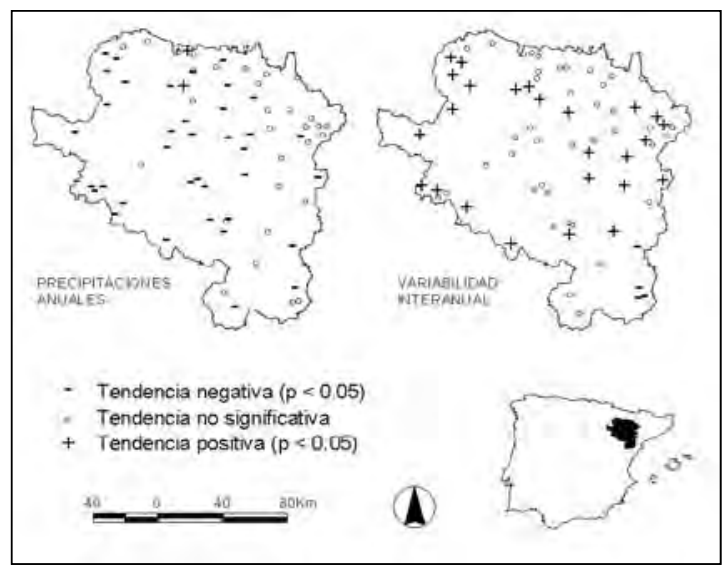

Figura 2.- Distribución espacial de la tendencia de precipitación anual, y del coeficiente de variación móvil anual en el sector central de la depresión del Ebro.

En la Tabla 7 se muestra la matriz de cruce y las probabilidades de transición empíricas entre ambas tendencias.

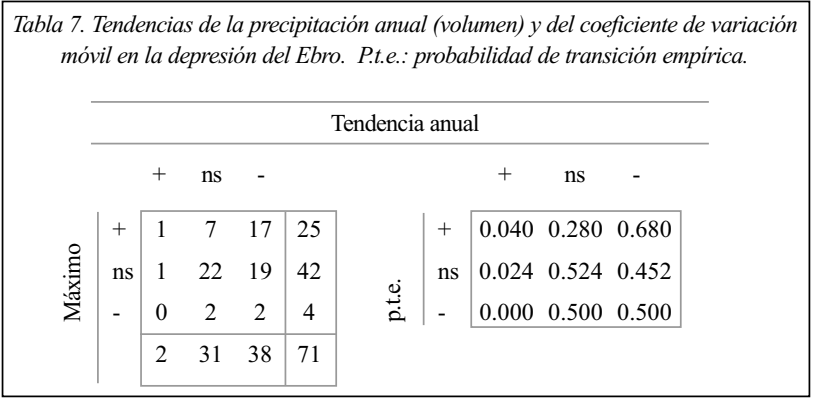

Los resultados del análisis de independencia, asociación, y simetría se muestran en la Tabla 8 . 


\begin{tabular}{|c|c|c|c|c|c|c|c|}
\hline \multicolumn{8}{|c|}{$\begin{array}{c}\text { Tabla 8. Resultados de las pruebas de independencia y simetría entre las tendencias } \\
\text { del volumen anual y de la variabilidad. Sector central de la depresión del Ebro. Se } \\
\text { incluye el CC y su porcentaje sobre el máximo (\% CCmax). Los signos (I, I) indican } \\
\text { la diagonal principal sobre la que se aplica la prueba de simetría, que nos indica la } \\
\text { dirección predominante del cambio. n.s.: no significativo. A (vol) es la tendencia en } \\
\text { el volumen anual de precipitación. }\end{array}$} \\
\hline & \multicolumn{4}{|c|}{ Independencia } & \multicolumn{3}{|c|}{ Simetría } \\
\hline & \multicolumn{4}{|c|}{ g.1. $(3-1)^{2}$, crit. 9.48} & \multicolumn{3}{|c|}{ g.l. $(3 \times(3-1)) / 2$, crit. 7.815} \\
\hline Comparación & $-^{2}$ & $\mathrm{p}$ & $\mathrm{CC}$ & $\% \mathrm{CC}_{\max }$ & $-^{2}$ & $\mathrm{p}$ & Diagonal \\
\hline A (vol) - Variabilidad & 4.979 & n.s. & - & - & 6.21 & n.s. & / \\
\hline
\end{tabular}

En el sector central de la depresión del Ebro la distribución espacial de las tendencias del volumen y de la anomalía de la precipitación anual parecen ser de carácter aleatorio $\left(\chi^{2} 4.979\right.$, n.s.). Por otra parte, las transiciones entre signos de ambas tendencias producen una matriz simétrica en la que las asociaciones entre signos no son directas.

En consecuencia, no existe asociación entre las tendencias anuales y su variabilidad, lo que origina un escenario espacialmente muy impredecible en el supuesto de que tales tendencias se mantengan en el futuro.

En un ambiente de elevada variabilidad pluvial como el analizado, el aumento de la variabilidad y de los volúmenes (que no es el caso) sugeriría que las precipitaciones entre años consecutivos estarían sometidas a fuertes variaciones, y en el tiempo los años húmedos serían seguidos de años secos. La aleatoriedad encontrada en el solape de los signos de la tendencia del volumen anual y de su variabilidad, sin embargo, sugieren unas condiciones de mayor carácter restrictivo, por cuanto pese a que en su totalidad las tendencias no se solapan de modo significativo, las transiciones entre variabilidades positivas y tendencias de volumen negativas agrupan a $1 / 4$ de los observatorios $(17$ casos, p.t.e. 0.680$)$.

\section{Discusión y Conclusiones}

El análisis temporal de los elementos climáticos es una tarea relevante en el contexto del cambio del clima. Si dicho análisis se realiza con un número adecuado de observatorios a lo largo de un número suficiente de años, de sus resultados se pueden deducir conclusiones importantes relacionadas con la dimensión espacial de dicho cambio.

Las dos premisas necesarias para realizar un análisis espacial del cambio de los elementos del clima son la disponibilidad de períodos prolongados de registros y una elevada densidad de observatorios. Ambas premisas afectan de manera distinta a los diferentes elementos del clima. En principio se sugiere que el análisis de los registros de cualquier elemento debe realizarse con un período mínimo de observaciones que deberían coincidir con los períodos normales de la Organización Meteorológica Mundial (Hulme, 1992; Hulme et al., 1995; Moron et al., 1995). 
Respecto a la segunda cuestión, el comportamiento de los diferentes elementos del clima implica que su cambio en una región solamente se puede detectar cuando se emplea una densa red de observatorios (Groisman y Legates, 1994; Hulme, 1995). No conocemos umbrales mínimos de densidad espacial para aceptar que los resultados de una investigación se consideren válidos, aunque existe un cierto consenso por el que se considera que el número de observatorios necesarios para documentar cambios en las precipitaciones es de cinco veces más que los necesarios para poder constatar cambios en otros elementos más estables del clima, como las temperaturas o las presiones (Vinnikov et al., 1990). Por último, en regímenes pluviales convectivos, en cuyas condiciones los valores de precipitación de un observatorio se concentran en el tiempo y son representativos de un espacio muy reducido (Cosgrove y Garstang, 1995), además de cumplir con los requisitos previos se impone la cautela ante los resultados de cualquier análisis.

Ante cartografías de diferentes tendencias climáticas, es muy tentador asignar supuestas correspondencias entre tendencias (anuales y estacionales, por ejemplo), pero este procedimiento puede inducir a conclusiones erróneas. Por otra parte la naturaleza categórica del dato representado (el signo de tendencia) impone numerosas restricciones y posibilidades de análisis. Por tal razón, el solape de documentos espaciales de signos de tendencias necesita ser sometido a un análisis que confirme la aleatoriedad o asociación de los signos, análisis que contemple la naturaleza categórica del dato presentado.

En las áreas de estudio las tablas de contingencia permiten apreciar relaciones no aparentes inicialmente. En la Comunidad Valenciana el análisis permite descubrir una relación doble entre las tendencias anuales y las del máximo evento diario; por su parte en el sector central del valle del Ebro la relación encontrada entre las tendencias de precipitación anual y su variabilidad sugiere que los escenarios futuros, si se mantienen las pautas descritas, estarán sometidos a una mayor imprecisión y aleatoriedad.

\section{Agradecimientos}

Estudio financiado por CICYT (REN2002-01023/CLI y BSO2002-02743), Diputación General de Aragón-FSE (DGA P003-2001) y Unión Europea (AQUADAPTEVK1-CT-2001-00104).

\section{Bibliografía}

Brunetti M., Maugeri M. y NANni T. (2001). Changes in total precipitation, rainy days and extreme events in northeastern Italy. International Journal of Climatology 21, 861-871.

Clark W.A.V. y Hosking P.L. (1986). Statistical methods for geographer. Chisterter, John Wiley \& Son, 518 pp.

Cosgrove C.M. y GARSTANG M. (1995). Simulation of rain event from raingauge measurements. International Journal of Climatology, 15, 1021-1029.

De Luís M. (2000). Estudio espacial y temporal de las tendencias de lluvia en la Comunidad Valenciana (1961-1990). Logroño, Ed. Geoforma, 112 pp.

De Luís M., Raventós J., González-Hidalgo J.C., SÁnchez J.R. y Cortina J. (2000). 
Spatial analysis of rainfall trends: a case study in Valencia Region (E Spain). International Journal of Climatology, 20: 1451-1469.

Downie N.M. y HeAth R.W. (1986). Métodos estadísticos aplicados. México D.F., Harla, ( $5^{\mathrm{a}}$ ed. in spanish), $379 \mathrm{pp}$.

Easterling D.R., Meehl G.A., Parmsan C., Chagnon S.A., Karl T.R. y Mearns L.O. (2000). Climate extremes: observations, modelling and impacts. Science, 249: 20682074.

GonzÁlez Hidalgo J.C., De Luís M., Raventós J. y SÁnchez Montahud J.R. (2001). Spatial distribution of seasonal rainfall trends in a western mediterranean area. International Journal of Climatology, 21: 843-860.

González Hidalgo J.C., de Luís M., Stepánek P., Raventós J. Y CuAdrat J.M. (2002a). Reconstrucción, estabilidad y proceso de homogeneizado de series de precipitación en ambientes de elevada variabilidad pluvial. En La información climática como herramienta de gestión ambiental (J.M. Cuadrat, S.M. Vicente y M.A. Saz, Eds). AGE - Universidad de Zaragoza, 47-57.

González Hidalgo J.C., Vicente S.M., de luis M., Stepánek P., Cuadrat J.M., RaVEntós J. y SÁnchez Montahud J.R. (2002b). Reconstrucción de registros pluviales y creación de una base de datos mensuales en la vertiente mediterránea española. En El Agua y el clima ( J.A. Guijarro, M Grimalt, M. Laita y S. Alonso). Publicaciones AEC, Serie A, No 3, 219-228.

Groisman P.Y. y Legates D.R. (1994). The accuracy of United States precipitation data. Bulletin American Meteorological Society, 75: 215-227.

Hulme M. (1992). Rainfall changes in Africa: 1931-1960 to 1961-1990. International Journal of Climatology, 12, 685-689.

Hulme M. (1995). Estimating global changes in precipitation. Weather, 50, 34-42.

Hulme M., Conway D., Jones P.D., Jiang T., Barrow E.M. y Turney C. (1995). Construction of a 1961-1990 European climatology for climate change modeling and impact applications. International Journal of Climatology, 15, 1333-1363.

KARL T.R. Y KNIGHT R.W. (1998). Secular trends of precipitation amount, frequency and intensity in the USA. 